Mens

revue d'histoire intellectuelle de l'Amérique française

David Karel, Peinture et société au Québec I (1603-1948), Québec, Les Éditions de l'IQRC et Les Presses de l'Université Laval, en collaboration avec le Musée national des beaux-arts du Quélbec, 2005. 160 p.

\title{
Louise Vigneault
}

Volume 7, numéro 1, automne 2006

URI : https://id.erudit.org/iderudit/1024230ar

DOI : https://doi.org/10.7202/1024230ar

Aller au sommaire du numéro

Éditeur(s)

Centre de recherche en civilisation canadienne-française

ISSN

1492-8647 (imprimé)

1927-9299 (numérique)

Découvrir la revue

Citer ce compte rendu

Vigneault, L. (2006). Compte rendu de [David Karel, Peinture et société au Québec I (1603-1948), Québec, Les Éditions de l'IQRC et Les Presses de

l'Université Laval, en collaboration avec le Musée national des beaux-arts du Québec, 2005. 160 p.] Mens, 7(1), 157-161. https://doi.org/10.7202/1024230ar 


\section{David Karel, Peinture et société au Québec I (1603- 1948), Québec, Les Éditions de l'IQRC et Les Presses de l'Université Laval, en collaboration avec le Musée national des beaux-arts du Québec, 2005. $160 \mathrm{p}$.}

L'ambition de l'ouvrage de David Karel consiste à fournir quelques éléments de réponse à la question du développement de la peinture dans le contexte social québécois, et ce, des tout débuts de la colonisation (1603) à la fin de la Seconde Guerre mondiale (1948). Au premier regard jeté sur cette plaquette, le projet peut sembler plus qu'ambitieux, compte tenu de l'étendue de la période historique couverte. L'éditeur avise toutefois le lecteur que, suivant les objectifs de la collection « Explorer la culture » des Éditions de l'IQRC, cet essai vise avant tout à "présenter une description d'ensemble mais succincte de l'évolution du secteur considéré, dans un langage accessible à tous les lecteurs désireux de s'y initier sans rechercher un savoir encyclopédique ou spécialisé » (jaquette verso). À titre de spécialiste invitée à évaluer cet ouvrage de référence "non spécialisé », j'ai donc éprouvé, en toute honnêteté, quelques difficultés à choisir un angle critique susceptible de rendre justice à la fois au lectorat ciblé et aux universitaires qui liront ce compte rendu...

L'ouvrage n'a donc d'autre ambition que de fournir un panorama des principaux facteurs de transformation des initiatives artistiques, en considérant les problèmes de rattrapage culturel, les efforts d'autonomisation et la " conquête d'originalité ». Il apporte d'abord un éclaircissement sur les premiers desseins de mise en image de la réalité du Nouveau Monde, depuis les entreprises d'évangélisation qui contrastaient alors avec la tradition iconoclaste protestante, en passant par les tentatives pragmatiques de Champlain de transmettre, au moyen d'illustrations, ses observations sur la na- 
ture, les données sur les cultures autochtones et les premiers établissements de la Nouvelle-France. Il est intéressant, par exemple, d'apprendre qu'à son arrivée, il avait déjà illustré des récits de voyage aux Antilles et au Mexique, avant d'illustrer ses récits de voyage au Canada de 1604 à 1612. Même si on débat encore aujourd'hui de la paternité de ces œuvres, elles ont néanmoins participé aux premières tentatives de rendre compte d'une vision soi-disant objective de la réalité du Nouveau Monde. Par la suite, les peintres missionnaires dont Claude François dit Frère Luc, Hugues Pommier, Claude Chauchetière - considéraient le langage pictural comme un instrument efficace de l'enseignement religieux, bien que le public susceptible d'apprécier ces œuvres à leur juste valeur fut longtemps des plus restreints. Le père jésuite Jean Pierron (1631-1700) valorisait d'ailleurs le langage visuel au détriment de celui de la parole comme instrument de communication de la foi, en raison de sa plus grande efficacité auprès des nations autochtones et des plus résistants "qui se fermoient avec les doits les oreilles» (p. 16). L'auteur rappelle ensuite que, sous le Régime anglais, la pratique picturale, qui ne visait pas encore l'originalité, évoluait entre la copie, l'adaptation et les initiatives personnelles. On voyait poindre, toutefois, une première prise de conscience d'une identité distincte et la nécessité de la construire. Il est heureux que l'auteur ait pris soin de mentionner le travail de l'artiste huron-wendat Zacharie Vincent, qui avait reçu une formation sous le tutorat d'Antoine Plamondon et qui avait su profiter de la popularité de la thématique autochtone et de celle de la " disparition de la race » au lendemain de l'échec du mouvement patriote. Il en est de même pour l'importance accordée aux artistes migrants - dont Otto Jacobi - ou de passage, qui ont laissé leur marque et contribué à diffuser efficacement les courants étrangers. 
Karel relate les principaux défis auxquels étaient confrontés les artistes à partir du XIX ${ }^{\mathrm{e}}$ siècle, soit l'édification d'un marché, de réseaux d'échange et de diffusion, etc. On peut observer, par conséquent, que les productions picturales souffriront, de manière chronique, jusqu'au $\mathrm{XX}^{\mathrm{e}}$ siècle, de problèmes d'isolement, de conservation, de perfectionnement et de transmission. Malgré un souci d'éviter une ligne trop strictement chronologique, l'ouvrage souffre par moment d'un manque d'homogénéité quant à l'angle d'analyse retenu. Le lecteur spécialiste sera également agacé par les informations trop souvent incomplètes et rapidement expédiées. Si l'auteur maitrise les enjeux de l'art ancien, la section consacrée aux productions modernes, qui s'étend sur une vingtaine de pages tout au plus, accuse certains manques. On se limite, en fait, à une sélection des principaux courants d'avant-garde dont les enjeux sont énoncés trop sommairement. De plus, les critères de sélection des productions ne sont pas toujours explicites, tandis que certaines œuvres évoquées ne sont pas soumises à l'examen sociologique annoncé. Par exemple, plutôt que d'aborder la thématique florale de Marian Scott et l'influence de Georgia O'Keeffe, mieux aurait valu mentionner le rôle déterminant qu'elle a joué dans les actions politiques de gauche menées par son mari, Frank Scott, ou auprès de Norman Bethune, afin de rendre à l'art sa fonction sociale. L'auteur se contente - et en conclusion uniquement - de rappeler la manière dont les groupes linguistiques francophone et anglophone se sont partagé inégalement le paysage social. On se demande également pourquoi le travail de Jean Dallaire est évoqué et non celui des membres du mouvement automatiste et de Jean Paul Lemieux, dont le travail s'est pourtant avéré déterminant dans la construction d'une identité collective. L'auteur passe également sous silence le rôle des organisations, sociétés et institutions qui ont annoncé les bouleverse- 
ments sociaux de la Révolution tranquille, les tensions idéologiques qui ont divisé l'École du meuble et l'École des beauxarts de Montréal (lesquelles ont motivé les avant-gardes à réorienter sans cesse leurs objectifs), la question du mécénat (qui a pourtant joué un rôle structurant dans le milieu anglophone), ainsi que l'importance de la Société d'art contemporain mise sur pied par John Lyman en 1939, de même que ses initiatives d'informer le grand public et d'instaurer un réseau de diffusion et d'exposition parallèle.

Point intéressant : l'auteur a su toutefois cerner certaines filiations diachroniques, les héritages transmis par les générations successives d'artistes et ce, malgré les orientations idéologiques divergentes. Il rappelle, par exemple, la filiation entre Suzor-Coté, Ozias Leduc et Borduas quant à l'équilibre visé entre les mouvements de rayonnement et d'introspection, l'enracinement et l'universalisme. À propos du lien entre Leduc et Borduas, Karel rapporte une citation de ce dernier rendant hommage à son vieux maître : "Je lui dois de m'avoir permis de passer au pouvoir du rêve qui débouche sur l'avenir » (p. 78). L'auteur conclut finalement par une affirmation surprenante d'évidence : «Le Québec aussi, c'est l'Amérique ». On s'étonne que cet aveu n'ait pas mieux inspiré l'orientation de la démonstration, compte tenu de ce qui est annoncé dans l'introduction au sujet des enjeux d'autonomisation et d'originalité.

Quelques mots, en terminant, sur l'illustration de la couverture : sans critiquer le travail de Caroline Merola, cette image qui charrie les principaux stéréotypes du champ artistique (l'éternelle palette de couleur qui tient lieu de paysage en vertu d'un glissement métaphorique, dans lequel s'active l'artiste de chevalet de la tradition moderne pleinairiste) ne rend nullement justice au contenu socio-historique de l'ouvrage. Il aurait était préférable, à notre avis, de rendre plutôt hom- 
mage à certaines œuvres citées et rarement exposées, surtout que la question des droits d'auteur ne se pose plus dans le cas de l'art ancien. On en conclut donc que l'éditeur a sans doute craint que des reproductions d'œuvres canadiennes précédant le $\mathrm{XX}^{\mathrm{e}}$ siècle ne rebutent les lecteurs potentiels. Cette occultation semble ainsi perpétuer les préjugés séculaires quant au manque de popularité de l'art ancien québécois. Cet hommage aurait constitué une heureuse occasion de mettre en valeur ces productions auprès d'un plus vaste public.

Louise Vigneault Département d'bistoire de l'art et d'etudes cinématographiques Université de Montréal

Auguste Viatte. D'un monde à l'autre. Journal d'un intellectuel jurassien au Québec (1939-1949), vol. 2, Novembre 1942-août 1945. Avec une postface pour la période août 1945-1949. Édité et présenté par Claude Hauser. Sainte-Foy, Paris et Courrendlin, Les Presses de l'Université Laval, L'Harmattan et Éditions Communication Jurassienne et Européenne, 2004. 385 p.

Auguste Viatte constitue sans aucun doute un des pionniers, avec Albert Girard et Robert Cornevin, des études littéraires francophones. Son œuvre, dont on doit citer notamment l'Histoire littéraire de l'Amérique française (1954) et l'Histoire comparée des littératures francophones (1980), est en particilier caractérisée par une ambitieuse visée comparatiste dont les enjeux et les potentialités sont loin d'être épuisés et mériteraient une relecture attentive, d'autant plus que l'apport novateur d'Auguste Viatte semble un peu négligé, voire presque 